\title{
Randomised prospective parallel trial of therapeutic versus subtherapeutic nasal continuous positive airway pressure on simulated steering performance in patients with obstructive sleep apnoea
}

Melissa Hack, Robert J O Davies, Rebecca Mullins, Soo Jeon Choi, Sharron Ramdassingh-Dow, Crispin Jenkinson, John R Stradling

\begin{abstract}
Background-Obstructive sleep apnoea (OSA) impairs vigilance and may lead to an increased rate of driving accidents. In uncontrolled studies accident rates and simulated steering performance improve following treatment with nasal continuous positive airway pressure (NCPAP). This study seeks to confirm the improvement in steering performance in a randomised controlled trial using subtherapeutic NCPAP as a control treatment.

Methods-Fifty nine men with OSA (Epworth Sleepiness Score (ESS) of $\geqslant 10$, and $\geqslant 10 / \mathrm{h}$ dips in $\mathrm{SaO}_{2}$ of $>4 \%$ due to $\mathrm{OSA}$ ) received therapeutic or subtherapeutic NCPAP $\left(\approx 1 \mathrm{~cm} \mathrm{H} \mathrm{H}_{2} \mathrm{O}\right)$ for one month. Simulated steering performance over three 30-minute "drives" was quantified as: standard deviation (SD) of road position, deterioration in SD across the drive, length of drive before "crashing", and number of off-road events. The reaction times to peripheral target stimuli during the drive were also measured.

Results-Subtherapeutic NCPAP did not improve overnight $>4 \% \mathrm{SaO}_{2} \mathrm{dips} / \mathrm{h} \mathrm{com}$ pared with baseline values, thus acting as a control. The SD of the steering position improved from 0.36 to 0.21 on therapeutic NCPAP, and from 0.35 to 0.30 on subtherapeutic NCPAP $(p=0.03)$. Deterioration in $\mathrm{SD}$ of the steering position improved from 0.18 to $0.06 \mathrm{SD} / \mathrm{h}$ with therapeutic NCPAP and worsened from 0.18 to 0.24 with subtherapeutic NCPAP $(p=0.04)$. The reaction time to target stimuli was quicker after therapeutic than after subtherapeutic NCPAP (2.3 versus 2.7 seconds, $p=$ 0.04).

Conclusions-Therapeutic NCPAP improves steering performance and reaction time to target stimuli in patients with OSA, lending further support to the hypothesis that OSA impairs driving, increases driving accident rates, and that these improve following treatment with NCPAP.
\end{abstract}

(Thorax 2000;55:224-231)

Keywords: sleep apnoea; driving; sleepiness
Obstructive sleep apnoea (OSA) is caused by airway occlusion during sleep, secondary to pharyngeal collapse. Each episode of collapse is usually terminated by a transient arousal from sleep which appears necessary to restore pharyngeal muscle tone and thus re-open the airway. Severely affected patients experience hundreds of obstructive episodes and brief arousals, with marked sleep disturbance leading to excessive daytime sleepiness. It has been suggested that this excessive sleepiness leads to falling asleep while driving and accounts for the higher accident rates seen in patients with OSA. ${ }^{1-13}$ Data are also available from driving simulators which suggest that patients with OSA are more impaired than normal control subjects. ${ }^{14-16}$

In 1981 Sullivan et $a l^{17}$ showed that splinting open the upper airway during sleep with continuous positive airway pressure (about $10 \mathrm{~cm} \mathrm{H}_{2} \mathrm{O}$ ) via a nasal mask (NCPAP) prevented the recurrent pharyngeal collapse. This was shown to reduce sleep fragmentation and improve sleep quality. ${ }^{18} \mathrm{~A}$ few studies have suggested a fall in the accident rate in patients treated with nasal CPAP, ${ }^{9}{ }^{10}$ and George et $a l^{19}$ have shown that impairment on a steering simulator in patients with OSA improves following treatment with nasal CPAP. ${ }^{19} \mathrm{Al}-$ though this study had normal control subjects, it did not have placebo treated controls. In a recent randomised controlled trial of nasal CPAP on the symptoms of OSA, using nasal CPAP at subtherapeutic pressures to provide a control arm, we found large treatment responses but also a significant placebo effect. ${ }^{20}$ This makes it particularly important to show that any improvements in performance seen on nasal CPAP are real and are not due to a placebo effect. We have therefore performed a randomised, parallel, prospective, double blind, controlled trial of the effects of nasal CPAP on simulated steering performance in patients with moderate to severe OSA.

\section{Methods}

SUBJECTS

Patients were considered technically eligible for this trial if they had excessive daytime sleepiness, sleep apnoea, and were men aged between 30 and 75 years. Excessive daytime sleepiness was defined as an Epworth Sleepiness Score of $\geqslant 10 .{ }^{21}$ Obstructive sleep apnoea 
was defined as $\geqslant 10 / \mathrm{h}$ falls of $>4 \%$ in arterial oxygen saturation $\left(\mathrm{SaO}_{2}\right)$ during a sleep study, with confirmatory evidence that these were due to pharyngeal collapse. All eligible patients were entered into the study unless they decided in favour of an alternative treatment such as weight loss, tonsillectomy, or mandibular advancement device; needed urgent NCPAP because of associated respiratory failure or were about to lose their job due to sleepiness; declined to participate; or had a mental disability that made informed consent impossible. The patients recruited to this study came mainly from a previously reported randomised controlled trial of NCPAP which looked at sleepiness and self-reported health status as the primary end points. ${ }^{20}$ The driving simulator only became available part of the way through this first trial, and not all patients subsequently agreed to do this part of the study. Thus, following the end of the first trial, with identical entry criteria and investigation protocol, the study recruited 11 further subjects to provide sufficient numbers (based on an interim analysis $^{22}$ ) who agreed to do the steering tests before and after therapeutic or subtherapeutic treatment.

SLEEP STUDY

The presence of OSA was established by a one night sleep study, recording body movement and heart rate as markers of sleep disturbance with arterial oxygen saturation measurements $\left(\mathrm{SaO}_{2}\right)$ and snoring as markers of respiratory impairment (Visi-Lab Monitoring System, Stowood Scientific Systems, Oxford, UK). ${ }^{23}$ A video recording of the whole night was available to confirm that abnormalities seen were due to OSA. The severity of OSA was quantified from the number of $>4 \%$ falls in $\mathrm{SaO}_{2}$ per hour of study. This index predicts the severity of OSA symptoms and its response to treatment at least as well as other commonly used indices. ${ }^{23}$

STEERING SIMULATION

A steering simulator was developed based on the work of Land. ${ }^{24}$ The program was written in $\mathrm{Q}$ Basic and produces a computer derived image of the moving edges of a road that winds pseudo-randomly, white on black as in night driving. An image of the bonnet of the vehicle is portrayed at the bottom of the screen. The subject steers using a standard computer games steering wheel (Grandprix 1, Thrustmaster, Hillboro, Oregon, USA). The object of each test is to steer the centre of the vehicle as accurately as possible down the middle of the road for 30 minutes. The correct and realistic relationship between turning the wheel and the effect this has on car position had been carefully established. ${ }^{24}$ The road ahead is displayed in its entirety, or with either the far part missing (simulating driving in fog) or with the near part missing (forcing the driver to only use the advance information to place the car), fig 1 . The rationale for limited views of the road ahead has been published previously but, essentially, while driving the eyes move between the far road in order to estimate the
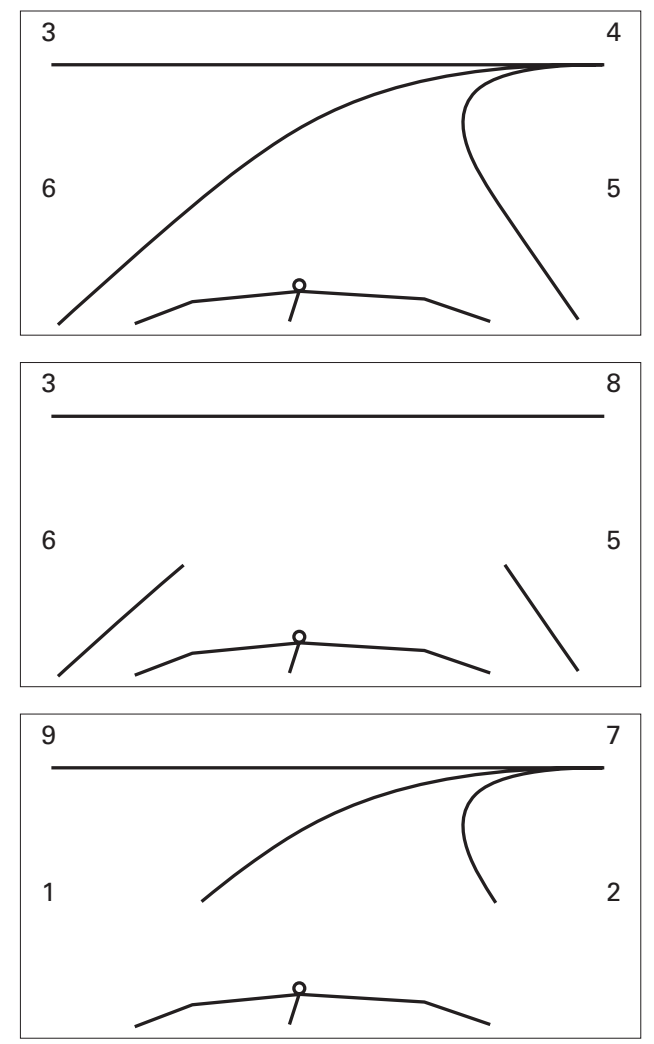

Figure 1 The three available views of the road ahead. The top panel shows the whole road, allowing use of both forward information (the approaching curve) and kerbside information to accurately place the car on the road. The middle panel shows the view having removed the forward information and leaves the driver dependent on the kerbside information alone. The bottom panel shows the view with only the forward information available.

coming curvature and the near road to ensure accurate placement in the lane, two separate processes requiring different skills. ${ }^{24}$ It had already been shown that patients with OSA perform badly on this simulator compared with matched control subjects. ${ }^{25}$

Single digits, one to nine, which change randomly at approximately $8-10$ second intervals, are displayed at each corner of the computer screen. While steering the subject is required to scan the four corners and identify a target digit each time it appears, approximately once per minute, by pressing a button on either side of the steering wheel. Inclusion of this visual search requirement produced a divided attention task. Before commencing their training all subjects received the same written and verbal instructions on the use of the simulator and the aim of the test. The training period to bring subjects to optimum performance was previously established to be two periods of six minutes and the test/retest reliability is satisfactory. ${ }^{25}$ Any residual learning effect will be controlled for through the use of a parallel design.

The initial 60 seconds of each full "drive" were excluded from the analysis to avoid including any "settling time". Position on the road relative to the centre was measured 20 times per second. The standard deviation (SD) of these data was used to give a measure of the variability of the steering error or the tendency 


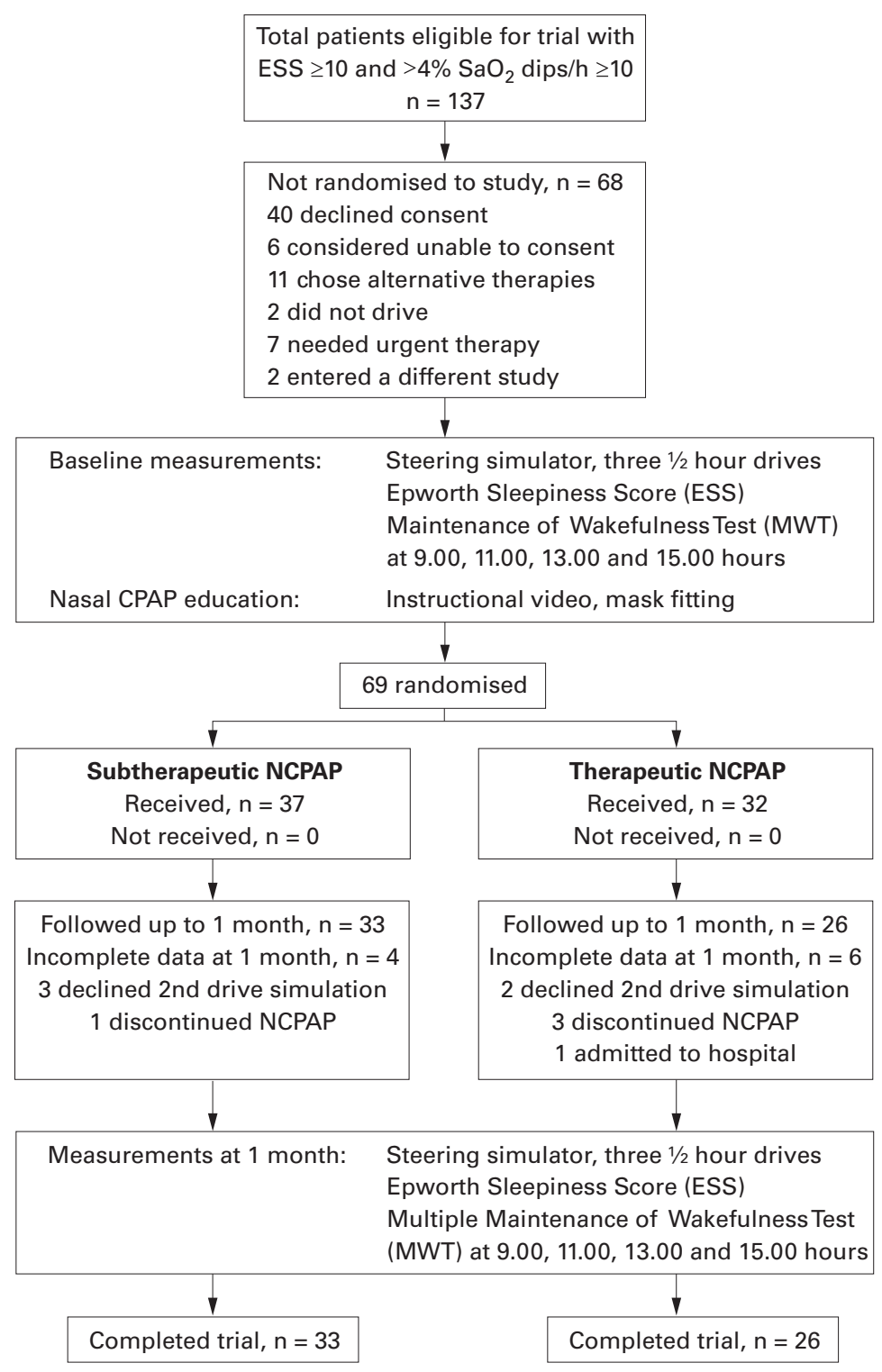

Figure 2 The study protocol and numbers at each stage of the trial.

to wander during the simulated drive. We also counted "off road" events, defined as occurring when the centre of the vehicle's bonnet crossed the lateral border of the road. The number of these events was quoted per hour of driving to allow for different length drives. The driver "crashed" if the vehicle remained off the road in this way for more than 15 seconds. In the event of a "crash" the analysis program terminated at this point and subsequent analysis excluded these last 15 seconds to avoid overestimation of overall tracking error. The SD was also calculated over sequential 10 second epochs and plotted against drive time to measure any deterioration across the drive. Mean reaction time for the detection of target digits was also measured for each drive.

SLEEPINESS

The Epworth Sleepiness Score (ESS) is a widely used index to quantify subjective sleepiness. ${ }^{21}$ It consists of eight questions about the tendency to fall asleep in situations of differing stimulation such as watching televi- sion or talking to someone. Each question is scored $0,1,2$, or 3 , indicating an increasing tendency to fall asleep in each situation; the total score can thus vary from 0 (no sleepiness) to 24 (extremely sleepy) with 9 being the upper limit of normal. ${ }^{26}$ Excessive daytime sleepiness was measured objectively using a modified maintenance of wakefulness test (MWT), a standard way to quantify this symptom. ${ }^{27}$ During the test subjects are asked to resist sleep while lying semi-recumbent in a darkened room for a maximum of 40 minutes on four occasions across a day $(9.00,11.00,13.00$ and 15.00 hours). During this time the subjects repeatedly tap a proximity detector with a finger in response to a dim, red, light emitting diode flashing regularly every three seconds. Sleep is defined as a failure to respond for 21 seconds (seven responses). After sleep onset the subject is awoken immediately. The average ability to resist sleep onset is quantified as the mean duration to sleep onset over the four challenges, and was one of the primary end points. This test gives results similar to those from the conventional MWT where sleep onset is verified by encephalographic, rather than behavioural, criteria. ${ }^{28}$

\section{INITIATION OF NCPAP AND SUBTHERAPEUTIC} NCPAP (CONTROL)

Initiation of NCPAP was performed as part of an overnight admission to hospital. All subjects were shown a video about NCPAP that included descriptions of the equipment and interviews with previous patients. A nurse specialist then selected the best fitting nasal mask and head gear for the patient and taught them how to use it. Subtherapeutic NCPAP was produced by providing a system for the patient identical in every way to therapeutic NCPAP except that the pressure at the mask was unlikely to be adequate to splint open the pharynx. The subtherapeutic pressure was achieved by using an NCPAP machine set to the lowest pressure possible $\left(\approx 3 \mathrm{~cm} \mathrm{H}_{2} \mathrm{O}\right)$ and by partially restricting airflow within the machine. In addition, six extra $4 \mathrm{~mm}$ holes were cut in the rubber collar of the connecting tubing at the mask end. These extra blow-off holes, in addition to the standard hole in the NCPAP mask, allowed more air to escape and kept the nasal pressure low while ensuring no $\mathrm{CO}_{2}$ reinhalation. Monitoring of the mask pressure using a home machine set to be subtherapeutic showed that, during ordinary quiet breathing, this arrangement provided a pressure that varied between +0.5 and $+1 \mathrm{~cm} \mathrm{H}_{2} \mathrm{O}$. By monitoring $\mathrm{SaO}_{2}$ over one night at home we established that this subtherapeutic pressure did not change the measured OSA severity. In a sample of 26 trial patients on subtherapeutic NCPAP, $>4 \% \mathrm{SaO}_{2}$ dips/h were 33 (5 to $95 \%$ confidence interval 6 to 72 ) compared with 29 (11 to 69) on the diagnostic night (difference not statistically significant).

PROTOCOL

The diagnosis and severity of OSA was established from a routine clinical sleep study and potential subjects were seen in the 
Table 1 Characteristics of patients participating in study

\begin{tabular}{|c|c|c|c|}
\hline & Subtherapeutic NCPAP $(n=33)$ & Therapeutic NCPAP $(n=26)$ & $\begin{array}{l}\text { p value } \\
\text { (between groups) }\end{array}$ \\
\hline Age (years) & $50(35$ to 64$)$ & $50(38$ to 68$)$ & 0.86 \\
\hline Weight $(\mathrm{kg})$ & $112(86$ to 160$)$ & $108(82$ to 141$)$ & 0.30 \\
\hline $\mathrm{BMI}\left(\mathrm{kg} / \mathrm{m}^{2}\right)$ & $34.0(27.0$ to 48.4$)$ & $32.2(26.3$ to 42.8$)$ & 0.24 \\
\hline Neck circumference $(\mathrm{cm})$ & $44.5(43.0$ to 50.9$)$ & $44.9(39.5$ to 50.8$)$ & 0.46 \\
\hline Time holding driving licence (years) & $29.5(14.5$ to 47.8$)$ & $31.5(11.3$ to 49.8$)$ & 0.62 \\
\hline$>4 \% \mathrm{SaO}_{2}(\mathrm{dips} / \mathrm{h})$ & $34.7(11.0$ to 70.1$)$ & $26.1(12.6$ to 55.5$)$ & 0.22 \\
\hline Maintenance of wakefulness test ( $\mathrm{min}$ ) & $20.2(7.2$ to 40$)$ & $21.2(6.3$ to 37.3$)$ & 0.75 \\
\hline Clinic Epworth Sleepiness score (ESS) & $15(11.2$ to 20.8$)$ & $15(10.0$ to 19.8$)$ & 0.32 \\
\hline SD of position on road & $0.35(0.15$ to 1.17$)$ & $0.36(0.15$ to 1.12$)$ & 0.92 \\
\hline SD deterioration $(\mathrm{SD} / \mathrm{h})$ & $0.18(-0.02$ to 2.67$)$ & $0.18(-1.14$ to 30.3$)$ & 0.58 \\
\hline Off-road events (no./h) & $34.8(0.9$ to 149$)$ & $17.8(0.4$ to 149$)$ & 0.59 \\
\hline Length of drive ( $\mathrm{min}$ ) & $27.6(10.9$ to 30.0$)$ & $24.8(7.6$ to 30.0$)$ & 0.57 \\
\hline Reaction time (s) & $2.8(1.7$ to 5.5$)$ & $2.8(1.8$ to 4.9$)$ & 0.59 \\
\hline
\end{tabular}

Values are medians (5th to 95 th centiles).

outpatient department to discuss the results. If patients fulfilled the entry criteria (ESS $\geqslant 10$ and $>4 \% \mathrm{SaO}_{2}$ dips/h $\geqslant 10$ ), then NCPAP was discussed along with other potential therapies where appropriate (fig 2). If the patient agreed to try NCPAP they were asked to enter the trial. It was explained that the study was comparing two different NCPAP pressures for the treatment of OSA and that we wanted to see which was the more effective. The extra tests and time involved in this study were explained. If the patients agreed, they were booked for two appointments 29 days apart. The first of these appointments was within five weeks of the outpatient visit and thus, for each patient, the trial period was completed within a maximum of nine weeks of recruitment. This was within the waiting time for routine non-research provision of NCPAP in our hospital.

Patients were admitted on the first day of the study for measurements by the research nurses of sleepiness (ESS and MWT) as well as their performance on the steering simulator during three 30 minute "drives" across the day, with the three different views of the road ahead, in random order. The tests were done at the same time of day on each occasion $(11.00,12.00$ and 14.00 hours) with the initial training period prior to the first "drive" on each day. That night the patients received an introduction to NCPAP from a nurse specialist, followed by randomisation to therapeutic or subtherapeutic NCPAP that was performed after this period of instruction. The DeVilbiss Horizon auto NCPAP machine was either set to autotitrate ${ }^{29}$ or to stay at its baseline pressure all night, and the connecting tube either did or did not have extra blow-off holes. A balanced randomisation schedule with a series of numbered opaque sealed envelopes was used, with the stratification dependent on the two entry criterianamely, severity of sleepiness (ESS) and severity of OSA $\left(>4 \% \mathrm{SaO}_{2}\right.$ dips $\left./ \mathrm{h}\right)$. The sealed envelopes were prepared in advance of the trial by CJ who was not involved in the treatment allocation.

The following morning the study subjects were sent home with an NCPAP machine set either at the pressure effectively controlling their OSA or at a pressure of $1 \mathrm{~cm} \mathrm{H}_{2} \mathrm{O}$, according to their randomisation the night before. In line with our usual clinical practice, all the patients were given telephone access to specialist nurses for advice on any problems experienced in the early stages of the treatment.

At four weeks patients were readmitted for a repeat of the daytime assessments of sleepiness and steering performance. The time clocks on the NCPAP machines were read and average nightly use over the month calculated. Thus, this was a parallel study involving random allocation to therapeutic or subtherapeutic NCPAP with assessments before and after one month of treatment.

\section{MASKING}

Patients were told that we were comparing two NCPAP pressures in order to find out which was the more effective in controlling their symptoms, and that one might be more effective than the other. Since they had never experienced NCPAP before, there was no reason for them to realise that the lower pressure might prove to be subtherapeutic. Combined with the parallel design, it was therefore extremely unlikely that the subjects would behave differently towards the two pressures in a way that would invalidate the masking. The research nurses (RM and SR) who measured the ESS, MWTs, and the steering performance were unaware of which NCPAP pressure the patient had received. In addition, they were not involved with the provision of NCPAP, patient management, telephone support, or equipment maintenance. This study was therefore effectively double blinded, despite the physical nature of the treatment.

The protocol was approved by the Central Oxford research ethics committee (number 96.127).

\section{ANALYSIS OF DATA}

The outcome measures were the derivatives from the mean of the three 30 minute drives on the steering simulator described above: standard deviation of steering position plus its deterioration with time, number of off-road events, length of drive before "crashing", and the average reaction time to peripheral targets. All data were entered onto a PC and analysed with the SPSS statistical package (SPSS UK Ltd, Surrey, UK). All values are median with 5 th to 95th centiles as most of the measures are not normally distributed. The statistical significance of changes following treatment was 


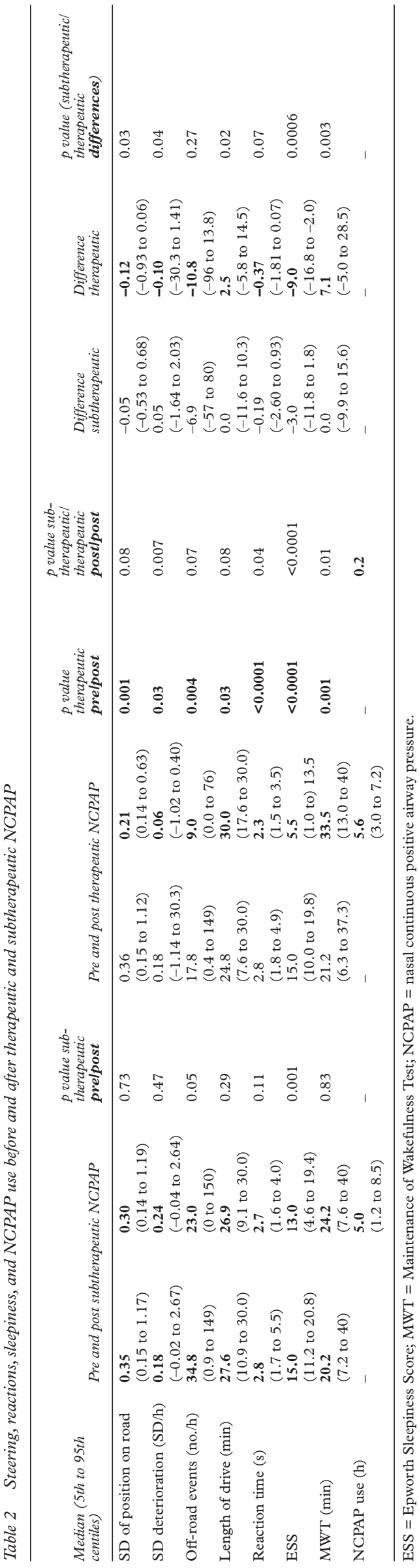

assessed in three ways in order to explore the consistency of any differences found between the groups. Firstly, the individual results before and after both subtherapeutic and therapeutic NCPAP were explored to provide information about the individual effects of each treatment arm and, in particular, the presence of any "placebo effect" (Wilcoxon matched pairs signed ranks test, pre/post columns in table 2). Secondly, the changes in the outcome measures due to treatment with either subtherapeutic or therapeutic NCPAP were compared (Wilcoxon rank sum tests, last column in table 2 ). Finally, the actual post-treatment outcome measures in the two groups were compared (Wilcoxon rank sum tests, post/post columns in table 2). This latter analysis is the severest test of any treatment effects seen in randomised controlled trials since it does not control for any baseline differences in the two groups and essentially determines whether there is any significant difference between the two groups after the treatment period.

\section{Results}

During the period of recruitment (October 1997 to March 1999) 137 patients were eligible for the study. Figure 2 shows the numbers completing each stage. Sixty eight subjects were not admitted: 40 refused, usually because of the extra time the study involved (two whole days and an extra night) or excessive travelling distances; six were considered too mentally impaired to give reliable informed consent; 11 chose alternative therapies or felt their symptoms did not warrant such an intrusive treatment as NCPAP; two did not drive; seven were considered to need urgent treatment, either because they were in respiratory failure or because of imminent job loss due to sleepiness and inability to drive; two were entered into a different study. Ten patients were not restudied at the one month follow up, usually due to the discontinuation of NCPAP or reluctance to repeat the study measurements. This left 33 subjects in the subtherapeutic group and 26 in the therapeutic group with complete data available for analysis.

Table 1 shows the similar baseline characteristics of the two groups. Table 2 shows the data for the measures of steering performance and reaction to targets before and after both subtherapeutic and therapeutic NCPAP. Also shown are the objective and subjective sleepiness results. Subtherapeutic NCPAP had no significant effect on the measures of steering (except for off-road events, $p=0.05$ ), reaction time, or MWT, although it had a significant effect on the subjective ESS ( $p=0.001)$. Therapeutic NCPAP produced significant improvements in the measures of steering and sleepiness compared with subtherapeutic NCPAP, although in one of the three analyses usual statistical significance was sometimes not quite reached. Figure 3 is an example of the steering performance before and after therapeutic NCPAP. Even for the off-road events, which are due to gross steering errors where the car is already half over the kerb, there is a trend in favour of therapeutic NCPAP. The use 

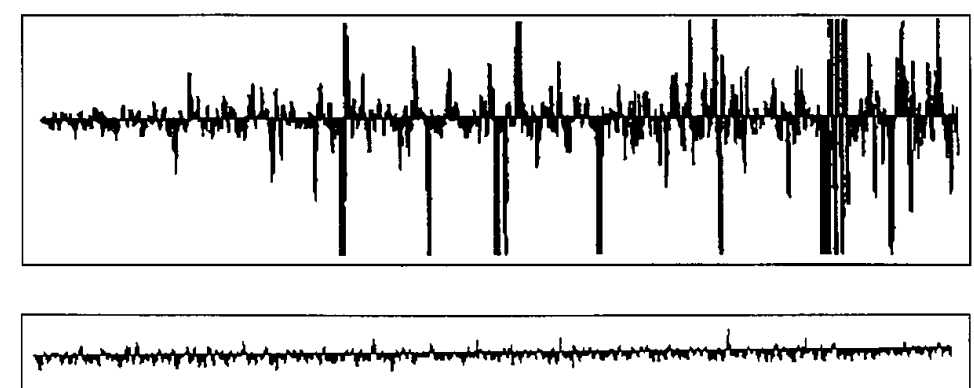

Figure 3 Example of the performance on the steering simulator of a patient with OSA before and after therapeutic NCPAP. The tracings run from left to right and represent the amount of wander about the middle of the curving road over a period of 30 minutes; the vertical axis is arbitrary. Note the overall better performance after NCPAP and the absence of deterioration in performance over the 30 minute period.

of NCPAP by the subtherapeutic group was 36 minutes per night less than that of the therapeutic group, although this did not reach statistical significance.

\section{Discussion}

This study has shown that, compared with control, therapeutic NCPAP improves measures of steering performance and response to target stimuli. In addition, in both the pretreatment groups and also the post subtherapeutic group, there was clear deterioration in steering performance across the 30 minute simulation periods which was virtually abolished by treatment with therapeutic NCPAP. This was also reflected in the improved ability of the post-therapeutic group to stay on the road longer and have fewer off-road events. All the results are internally consistent and overall provide clear evidence in patients with OSA of a significant effect of therapeutic NCPAP on steering performance, using a simulator that tests visual processes involved in real driving, as well as the reaction time to peripheral target stimuli. There was a small but significant placebo effect on the number of off-road events, and this shows the importance of placebo controls in this area.

This study used subtherapeutic levels of NCPAP to produce a control treatment as similar to normal NCPAP as possible, without producing any measurable improvement or deterioration in OSA severity. There had been concerns over the safety of subtherapeutic NCPAP, ${ }^{30}$ but our particular design prevented patients from reinhaling $\mathrm{CO}_{2}$ or experiencing negative inspiratory pressures, and is being used successfully elsewhere. ${ }^{31}$ Because there is a perceptible difference between the subtherapeutic pressure $\left(\approx 1 \mathrm{~cm} \mathrm{H}_{2} \mathrm{O}\right)$ and a therapeutic pressure, we recruited patients naive to NCPAP and did not employ a crossover strategy. There was no reason for the subjects to suspect they might be on an ineffective pressure, although the slightly lower NCPAP usage by the subtherapeutic group might have been the result of less enthusiasm due to its lack of efficacy. We were careful to ensure blinding of both the patients and the research nurses who carried out the measures of sleepiness and supervised the simulated driving performance. We are therefore confident that this trial was as double blind as is possible with a physical therapy.

Specific entry criteria defining OSA severity were required for this study. However, the correlations between conventional sleep study measures of OSA severity and sleepiness (or its subsequent improvement with NCPAP) are not good and rarely exceed 0.5 even in the most careful studies. ${ }^{23}{ }^{32}$ This is probably because the currently available sleep study indices of OSA severity do not measure all aspects of sleep fragmentation and therefore cannot predict accurately the effects on daytime sleepiness or performance. There are many intermediary steps between obstructive respiratory events at night and symptoms of excessive sleepiness during the day. Events other than apnoeas can lead to arousal (such as hypopnoeas and obstructive snoring), the degree of arousal required to clear the airway varies, ${ }^{33}$ obstructive events may cluster or be spread out, extra sleep may be taken during the day, and differing daytime activities will alter the symptomatic impact of sleep fragmentation. Measures of OSA severity include reductions in airflow at the nose and mouth, falls in $\mathrm{SaO}_{2}$, and various markers of recurrent arousal from sleep. Despite theoretical differences between these indices they are quite similar in their ability to predict excessive daytime sleepiness and the response of this symptom to treatment with $\mathrm{NCPAP}^{23}$ although, in absolute terms, values for $>4 \% \mathrm{SaO}_{2} \mathrm{dips} / \mathrm{h}$ are a little lower than the equivalent apnoea/hypopnoea indices. We identified patients for this study simply using the number of $>4 \% \mathrm{SaO}_{2}$ dips per hour since limited sleep study systems and oximeters are being used increasingly to quantify OSA rather than full polysomnography, with the evidence supporting this transition. ${ }^{23}$ Although we had to set an arbitrary threshold based on OSA severity for the entry criteria, sleep units see patients with OSA who have $>4 \% \mathrm{SaO}_{2}$ dip rates lower than the entry criteria for this study who nonetheless may respond to NCPAP with objective improvements in sleepiness.

The steering simulator used in this study has been designed to engage two of the visual processes known to help drivers position their cars in the right place on the road. ${ }^{24}$ Thus it is likely that this simulator is testing visual processes involved in real driving which are further challenged by the addition of peripheral target detection, making it a divided attention task, again similar to real driving. However, there are many other aspects of driving not tested in this simulator and any relationship to real driving accidents will require separate validation.

Sleep deprivation in normal subjects has been shown by many other groups to produce reduced performance in both cognitive and vigilance based tests. ${ }^{34-38}$ In addition, sleep deprivation produces deterioration of vigilance across a task, particularly if it is unstimulating. ${ }^{39}$ There is some evidence that patients with OSA may have cognitive impairment in addition to simple impairment of vigilance. ${ }^{32} 40$ This could be secondary to many years of sleep fragmentation or be a consequence of the nocturnal hypoxia often present 
in patients with significant OSA. ${ }^{41}{ }^{42}$ Although the vigilance may return rapidly to normal following NCPAP therapy, ${ }^{20}$ cognitive function may take longer or possibly never return to normal. ${ }^{42}{ }^{43}$ The relative contribution of poor vigilance or impaired cognition to impaired driving performance in patients with OSA may be difficult to apportion. Because of the evidence that there are persisting cognitive defects in patients with OSA following treatment, it will be important to establish if driving abilities return fully to normal. Although the therapeutic group improved considerably on treatment at one month, the levels of performance did not quite fall to figures obtained for a matched control group from an earlier study (SD of the driving position 0.21 versus 0.15 , reaction time 2.3 versus $1.8 \mathrm{~s}$, off-road events 9.0 versus $0 / \mathrm{h}$, post NCPAP treatment and normal controls, respectively). ${ }^{25}$ Further studies will be necessary to establish if there is any residual impairment, despite the abolition of sleep fragmentation, after longer periods on treatment. We did not find a significant correlation between the improvement in sleepiness (ESS or MWT) and the improvement in steering performance in the 26 patients receiving therapeutic treatment, perhaps suggesting that sleepiness and hence reduced vigilance may not be the only factor that impairs steering performance and responds to NCPAP treatment.

The levels of improvement seen in this study following NCPAP are similar to those seen in the uncontrolled studies of George et al. ${ }^{19}$ Although there was a clear placebo effect on the subjective measures of sleepiness (ESS), ${ }^{20}$ we found no such effect on the objective measure of sleepiness (MWT) or on steering performance (except for off-road events).

This randomised controlled trial has confirmed the benefits found in previous uncontrolled studies of NCPAP on steering performance and the response time to peripheral target stimuli. The effects are significant and lend support to the hypothesis that higher car accident rates in patients with OSA, and their reduction on treatment, are real observations. This provides further evidence for the importance of treating this group of patients, something that has been called into question in the recent past. ${ }^{44}$

The study was supported by the NHS Executive (Anglia and Oxford), grant number HSR/UOX/0296/72 and the British Lung Foundation, grant number 98/11. We thank all the Oxford Sleep Unit staff who willingly cooperated in the execution of this trial.

1 George C, Nickerson PW, Hanly PJ, et al. Sleep apnoea patients have more automobile accidents. Lancet 1987;ii:447.

2 Findley LJ, Fabrizio M, Thommi G, et al. Severity of sleep apnea and automobile crashes. $N$ Engl F Med 1989;320: $868-9$

3 Findley LJ, Unverzagt ME, Suratt PM. Automobile accidents in patients with obstructive sleep apnea. Am Rev Respir Dis 1988;138:337-40.

4 Findley LJ, Weiss JW, Jabour ER. Drivers with untreated sleep apnea. A cause of death and serious injury. Arch Intern Med 1991;151:1451-2.

5 Haraldsson PO, Carenfelt C, Diderichsen F, et al. Clinical symptoms of sleep apnea syndrome and automobile accidents. F Otorhinolaryngol Relat Spec 1990;52:57-62.

6 Cassel W, Ploch T, Peter JH, et al. Risk of accidents in patients with nocturnal respiration disorders. Pneumologie patients with nocturnal r.

7 Haraldsson PO, Carenfelt C, Tingvall C. Sleep apnea syndrome symptoms and automobile driving in a general population. $\mathcal{F}$ Clin Epidemiol 1992;45:821-5.
8 Wu H, Yan Go F. Self-reported automobile accidents involving patients with obstructive sleep apnea. Neurology 1996;46:1254-7

9 Cassel W, Ploch T, Becker C, et al. Risk of traffic accidents in patients with sleep-disordered breathing: reduction with nasal CPAP. Eur Respir f 1996;9:2606-11.

10 Krieger J, Meslier N, Lebrun T, et al. Accidents in obstructive sleep apnea patients treated with nasal continuous positive airway pressure: a prospective study. The Working Group ANTADIR, Paris and CRESGE, Lille, France. Association Nationale de Traitement a Domicile des Insuffisants Respiratoires. Chest 1997;112:1561-6.

11 Engleman HM, Hirst WS, Douglas NJ. Under reporting of sleepiness and driving impairment in patients with sleep

12 Teran-Santos J, Jimenez-Gomez A, Cordero-Guevara J, et al. The association between sleep apnoea and the risk of traffic accidents. N Engl f Med 1999;340:847-51.

13 Suratt PM, Findley LJ. Driving with sleep apnea. $N$ Engl f Med 1999;340:881-3.

14 Findley LJ, Fabrizio MJ, Knight $\mathrm{H}$, et al. Driving simulator performance in patients with sleep apnea. Am Rev Respir Dis 1989;140:529-30.

15 George CF, Boudreau AC, Smiley A. Simulated driving performance in patients with obstructive sleep apnea. $A m \mathcal{F}$ Respir Crit Care Med 1996;154:175-81.

16 George CF, Boudreau AC, Smiley A. Comparison of simulated driving performance in narcolepsy and sleep apnea lated driving performance in nart
patients. Sleep 1996;19:711-7.

17 Sullivan CE, Issa FG, Berthon-Jones M, et al. Reversal of obstructive sleep apnoea by continuous positive airway pressure applied through the nares. Lancet 1981;i:862-5.

18 Issa FG, Sullivan CE. The immediate effects of nasal continuous positive airway pressure treatment on sleep pattern in patients with obstructive sleep apnea syndrome. Electroencephalogr Clin Neurophysiol 1986;63:10-7.

19 George CFP, Boudreau AC, Smiley A. Effects of nasal CPAP on simulated driving performance in patients with obstructive sleep apnoea. Thorax 1997;52:648-53.

20 Jenkinson C, Davies RJO, Mullins R, et al. Randomised prospective parallel trial of therapeutic nasal continuous positive airway pressure (NCPAP) against sub-therapeutic NCPAP for obstructive sleep apnoea. Lancet 1999;353: $2100-5$

21 Johns MW. Daytime sleepiness, snoring, and obstructive sleep apnea. The Epworth sleepiness scale. Chest 1993;103: 30-6.

22 Hack MA, Davies RJO, Stradling JR. Randomised, sham placebo, parallel study of the effect of nasal continuous positive airway pressure (NCPAP) on steering performance in patients with obstructive sleep apnoea (OSA) - interim analysis. Thorax 1998;53(Suppl 4):A44.

23 Bennett LS, Langford BA, Stradling JR, et al. Sleep fragmentation indices as predictors of daytime sleepiness and nCPAP response in OSA. Am $\mathcal{F}$ Respir Crit Care Med and $\mathrm{nCPAP}$ respo

24 Land M, Horwood J. Which parts of the road guide steering? Nature 1995;377:339-40.

25 Juniper M, Hack MA, George CF, et al. Steering simulation performance in patients with obstructive sleep apnoea (OSA) and matched control subjects. Eur Respir f 2000 (in press).

26 Johns $M$, Hocking B. Daytime sleepiness and sleep habits of Australian workers. Sleep 1997;20:844-9.

27 Doghramii K, Mitler MM, Sangal RB, et al. A normative study of the maintenance of wakefulness test (MWT). Electroencephalogr Clin Neurophysiol 1997;103:554-62.

28 Bennett LS, Stradling JR, Davies RJO. A behavioural test to assess daytime sleepiness in obstructive sleep apnoea. 7 Sleep Res 1997;6:142-5.

29 Stradling JR, Barbour C, Pitson DJ, et al. Automatic nasal continuous positive airway pressure titration in the laboratory, patient outcomes. Thorax 1997;52:72-5.

30 Krieger J, Weitzenblum E, Monassier JP, et al. Dangerous hypoxaemia during continuous positive airway pressure reatment of obstructive sleep apnoea. Lancet 1983;ii: 1429-30.

31 Farre R, Hernandez L, Montserrat JM, et al. Sham continuous positive airway pressure for placebo-controlled studies in sleep apnoea. Lancet 1999;353:1154.

32 Cheshire K, Engleman H, Deary I, et al. Factors impairing daytime performance in patients with the sleep apnoeal hypopnoea syndrome. Arch Intern Med 1992;152:538-41.

33 Stradling JR, Pitson DJ, Bennett LS, et al. Variation in the arousal pattern following obstructive events in obstructive sleep apnea. Am f Respir Crit Care Med 1999;159:130-6.

34 Bonnet MH. Cognitive effects of sleep and sleep fragmentation. Sleep 1993;16:S65-7.

35 Bonnet MH. Sleep restoration as a function of periodic awakening, movement, or electroencephalographic change. Sleep 1987;10:364-73.

36 Glenville M, Broughton R, Wing AM, et al. Effects of sleep deprivation on short duration performance measures compared to the Wilkinson auditory vigilance task. Sleep 1978; 1:169-76.

37 Martin SE, Engleman HM, Deary IJ, et al. The effect of sleep fragmentation on daytime function. Am $\mathcal{F}$ Respir Crit Care Med 1996;153:1328-32.

38 Chugh DK, Weaver TE, Dinges DF. Neurobehavioral consequences of arousals. Sleep 1996;19:S198-201.

39 Wilkinson RT. Methods for research on sleep deprivation and sleep function. In: International psychiatry clinics, Vol 7, No. 2. Boston: Little, Brown and Co, 1970: 369-81. 
40 Naegele B, Thouvard V, Pepin JL, et al. Deficits of cognitive executive functions in patients with sleep apnea syndrome. executive functions in

41 Bedard MA, Montplaisir J, Richer F, et al. Nocturnal hypoxemia as a determinant of vigilance impairment in sleep apnea syndrome. Chest 1991;100:367-70.

42 Feuerstein C, Naegele B, Pepin JL, et al. Deficits of cognitive executive functions in patients with sleep apnea syndrome Acta Neurol Belg 1997;97:96-107.
43 Bedard MA, Montplaisir J, Malo J, et al. Persistent neuropsychological deficits and vigilance impairment in sleep apnea syndrome after treatment with continuous positive airways pressure (CPAP). I Clin Exp Neuropsychol 1993; 15:330-41.

44 Wright J, Johns R, Watt I, et al. Health effects of obstructive sleep apnoea and the effectiveness of continuous positive airways pressure: a systematic review of the research evidence. BMF 1997;314:851-60. 